\title{
A focus on paediatric obstructive sleep apnoea
}

This issue has two linked reviews on paediatric obstructive sleep apnoea (OSA) by Pabla and colleagues. ${ }^{1,2}$ This follows on from many articles in The Journal of Laryngology \& Otology on this perennially interesting topic, notably those concerning cardiopulmonary aspects of paediatric OSA, ${ }^{3}$ the assessment of paediatric OSA, ${ }^{4}$ tonsillotomy versus tonsillectomy, ${ }^{5}$ and the use of adenoidectomy alone. ${ }^{6}$ These two reviews cover such controversies as tonsillotomy versus tonsillectomy, anaesthetic considerations, postoperative monitoring, determination of when a threshold is reached at which treatment is required, and the wider medical aspects of sleep apnoea in children.

Polysomnography services for children in the average UK unit are limited, and using US criteria they would be rapidly swamped by demand, so only a minority of otolaryngologists use this routinely. Overnight oximetry is one of many screening methods, as are questionnaires, but is such screening adequate? The authors note that OSA in children should not be assumed to be the same qualitatively as in adults, with a 'good' or a 'bad' night (compared to 'usual') potentially distorting a test result and leading to possible under- or over-treatment.

Well-defined assessment criteria that link well with outcomes of treatment seem to be some way off, as is knowledge of the threshold at which significant neurocognitive, behavioural, metabolic or cardiac morbidity is likely to occur and so needs active intervention. This threshold may be lower than we currently believe. Is obesity part of the result of OSA, part of the cause of it, or both?

The increasing prevalence of obesity in childhood has now reached worrying levels (28 per cent of $2-15$ year olds were found to be overweight in
2015), so reviewing the medical aspects of paediatric sleep-disordered breathing is particularly topical. Similarly, the difficulty of dealing with a child after adenotonsillectomy, usually but not always obese, who still has significant sleep apnoea, is acknowledged and explored in these reviews. The authors draw from paediatric and other medical literature that is otherwise unlikely to be read by a general otolaryngologist. As with all useful reviews, there are more questions than answers.

EDWARD FISHER ROBIN YOUNGS

MUSHEER HUSSAIN JONATHAN FISHMAN Senior Editors

References

1 Pabla L, Duffin J, Flood L, Blackmore K. Paediatric obstructive sleep apnoea: can our identification of surgical candidates be evidence-based? J Laryngol Otol 2018;132:284-92.

2 Pabla L, Duffin J, Flood L, Blackmore K. Paediatric obstructive sleep apnoea: is our operative management evidence-based? J Laryngol Otol 2018;132:293-8.

3 Mahajan M, Thakur JS, Azad RK, Mohindroo NK, Negi PC. Cardiopulmonary functions and adenotonsillectomy: surgical indications need revision. J Laryngol Otol 2016;130:1120-4.

4 Sharma SD, Kanona H, Kumar G, Kotecha B. Latest trends in the assessment and management of paediatric snoring and sleep apnoea. J Laryngol Otol 2016;130:482-9.

5 Wood JM, Cho M, Carney AS. Role of subtotal tonsillectomy ('tonsillotomy') in children with sleep disordered breathing. J Laryngol Otol 2014;128(suppl 1):S3-7.

6 Reckley LK, Song SA, Chang ET, Cable BB, Certal V, Camacho M. Adenoidectomy can improve obstructive sleep apnoea in young children: systematic review and meta-analysis. J Laryngol Otol 2016;130:990-4. 